\title{
Digital Support for Indigenous Research Methodologies
}

\section{Kathleen Clapham}

University of Wollongong, Wollongong, Australia

kclapham@uow.edu.au

\section{Helen Hasan}

University of Wollongong, Wollongong, Australia

hasan@uow.edu.au

\section{Bronwyn Fredericks}

University of Queensland, Brisbane, Australia

\section{Dawn Bessarab}

University of Western Australia, Perth, Australia

\section{Peter Kelly}

University of Wollongong, Wollongong, Australia

\section{Valerie Harwood}

University of Sydney, Sydney, Australia

\section{Kate Senior}

University of Newcastle, Newcastle, Australia

\section{Marlene Longbottom}

University of Wollongong, Wollongong, Australia

\section{Elizabeth Dale}

University of Wollongong, Wollongong, Australia

\section{Abstract}

Research undertaken by outsiders into issues of concern to Aboriginal communities frequently ignores community culture and the knowledge embedded within Aboriginal communities. Methodologies are adopted which perpetuate the colonialist mindset of non-indigenous Australians leading to failed solutions to Aboriginal problems. This paper describes an Aboriginal-led community-based research project, exploring the role of Aboriginal Australians in caring for, and transforming, their own communities. It focuses on the roles that Information Systems can play when providing an accessible platform for Aboriginal voices. The authors conducted an in-depth case study of one Aboriginal Community Controlled Organisation (ACCO), the Illawarra Koori Men's Support Group (IKMSG). The research consisted of a social network analysis (SNA) of the inter-organisational links of the IKMSG; interviews and focus groups with members of the IKMSG and the co-design of their first website. The prominence of the IKMSG in the SNA maps suggests that its work in the community is highly respected and that the model produced by this research can act as a guide for success in other ACCOs. The findings have been used to develop a theoretical model of Aboriginal community engagement and intervention. This model can enable authentic outcomes to projects which address Aboriginal concerns and support the conduct of community-led research in Aboriginal communities.

Keywords: Indigenous Research Methodologies, Digital Support, Community Participation, Aboriginal Community Controlled Organisation, Social Network Analysis 


\section{Introduction}

Research into issues of concern to Aboriginal communities is frequently undertaken by 'outsiders' who are ignorant of the profound knowledge embedded within Aboriginal communities and indifferent to local community culture (Martin, 2008). The continuance of the deficit mindset, which focusses on problems rather than potential, contributes to the long history of failed solutions to 'Aboriginal problems' (Hollinsworth, 2013; Fforde et al., 2013; Fogarty, 2018; Gorringe et al., 2011). Many of the challenges faced by Indigenous communities throughout the world are perpetuated by the colonialist narrative (Smith 2012); this also persists throughout the research domain and shapes accepted research methodologies. Indigenous research methodologies have been developed to disrupt this narrative. Indigenous scholars such as Wilson (1998), Bessarab and Ng'andu (2010), Martin (2003), Smith (2012) and Walter and Moreton-Robinson (2010) demonstrate the efficacy of such methodologies when conducting research into Indigenous issues and where the findings of the research underpin decision-making impacting Aboriginal futures. The components of Indigenous research methodologies include the co-design of research, training community researchers and involving them in community research, and the use of relational data collection methods such as 'yarning' (Bessarab \& Ng' andu, 2010). These approaches result in community-led solutions to Indigenous challenges that are viable and sustainable (See for example Fredericks, 2016; Fredericks et al., 2011; Walker et al., 2013).

In this paper we propose that the application of suitable digital tools within such methodologies can support authentic data collection and analysis as well as the meaningful display of findings and dissemination of results to community. We support this proposition with findings from Aboriginal-led, community-based research which explored the role of Aboriginal Australians in caring for, and transforming, their own communities. The authors conducted an in-depth case study of one Aboriginal Community Controlled Organisation (ACCO) and the Illawarra Koori Men's Support Group (IKMSG), based in south eastern New South Wales (NSW). The study, part of a wider Caring for Community project, examined the positive impact of the IKMSG on the health and wellbeing of the Aboriginal men and families in the Illawarra region. Over the past decade, The IKMSG has provided placed-based culturally based programs that address issues of concern to Aboriginal men and their families (Ngarruwan Ngadju, 2017a). The research was conducted by a multidisciplinary team of Aboriginal and non-Aboriginal researchers, together with members of the IKMSG and other community stakeholders. The results of the study, presented in a report given to the community (Ngarruwan Ngadju, 2017b), show, firstly, how ACCOs can leverage the knowledge inherent in communities to build resilience and create better futures; and secondly, how real participation and inclusive decision making, provide the authority that enables change.

In this paper, we report on the suitability and efficacy of the digital tools adopted for the study and their potential support for Indigenous Research Methodology more generally. We argue that digital tools can (i) support many activities in the conduct of research in Aboriginal communities; (ii) enable authentic outcomes to projects which address Aboriginal issues and concerns; (iii) help in the management Aboriginal knowledge; and (iv) provide a platform for Aboriginal voices. 
Digital tools which consisted of a social network analysis (SNA) of the inter-organisational links of the IKMSG, were utilised to support data collection throughout this research project. This occurred in parallel with qualitative interviews and focus groups with members of the IKMSG, and the co-design of their first website. The findings from the IKMSG case study were used to develop a theoretical model of Aboriginal community engagement and interventions. The prominence of the IKMSG in the SNA maps suggests its work is highly respected in both Aboriginal and non-Aboriginal local organisations. The central position of IKMSG in the SNA maps provided authenticity to the model produced by this research so that it is widely accepted as a guide for designing programs in other ACCOs undertaking community programs with Aboriginal men.

We suggest that certain contemporary digital technologies are eminently suited to data collection and analysis using Indigenous research methodologies because these can be used to connect with, and be reflective of, the importance that Indigenous peoples place on relationality (Wilson, 1998). In our research, SNA is one such technology that can be used to assist with the analysis and depiction of vital relationships within networks of ACCOs and partner organisations. In the context of the partnership between local ACCOs and the university-based researchers, we also found that online forums such as Facebook, websites and blogs for knowledge transfer and for dissemination of the findings of the research, both inform community and provide an opportunity for their reflective comments, questions and suggestions.

\section{Background}

There is a substantial body of research in the disciplines of social work (Bessarab et al., 2014), education (Armstrong et al., 2012; Sarra, 2011; Vigo, 2017), health policy (Fogarty et al., 2018) and media studies (McCallum et al., 2012) that illustrate the negative consequences of the deficit mindset resulting from the colonialist narrative among non-indigenous Australians for the Aboriginal communities they supposedly aim to improve. The past two decades has witnessed an emergence of a body of scholarship that is led by Indigenous scholars from Canada, the United States of America, Australia and New Zealand and elsewhere. This has established a distinct Indigenous research paradigm (Wilson, 1998; Bessarab \& Ng'andu, 2010; Denzin \& Lincoln, 2008; Martin, 2003; Smith, 2012; Walter \& Moreton-Robinson, 2010; Williams et al., 2021). In her seminal work, Decolonising Methodologies, Maori academic Linda Tuhiwai Smith (Smith 2012) identifies the negative history associated with research and harmful impacts that Indigenous Peoples have experienced since colonisation. Australian Aboriginal academic Karen Martin (2003) identifies four principles that underlie the practice of research using Indigenous methodologies: Firstly, recognising Aboriginal worldviews, knowledge and realities as distinctive and vital to Aboriginal existence and survival, and serves as a research framework; secondly, honouring Aboriginal social mores as essential processes through which Aboriginal people live, learn and situate themselves in their own lands and in the lands of other Aboriginal peoples; thirdly, emphasising the social, linguistic, cultural, historical and political contexts which shape Aboriginal experience, lives, positions and futures; and fourthly, privileging the voices, experiences and lives of Aboriginal people and Aboriginal lands.

Work on specific data collection methods is more recent. In qualitative methods, yarning has been developed as a decolonising methodology in the work of Bessarab and others (Bessarab 
\& Ng'andu, 2010; Lin, Green, \& Bessarab, 2016; Walker et al., 2013). Decolonising ideas have also been applied within quantitative methods; Aboriginal scholars have developed Aboriginal statistics, using the terminology of data sovereignty to challenge the deficit narrative through the collection and analysis of numerical data (Walter \& Suina, 2018; Walter \& Andersen, 2013).

Many of these writings draw more broadly on critical social theories, for example Walter and Moreton-Robinson (2010). Indigenous Standpoint Theory (Foley, 2003; Moreton-Robinson, 1999; Nakata, 2007) and Indigenous Feminist Theory (Moreton-Robinson, 2000; Fredericks, 2009), provide new directions in the development of methodology which enables Aboriginal researchers to critically re-assess the corpus of knowledge written about them; and for nonindigenous researchers, it provides a framework for understanding their role, their potential, and their limitations within the power relations that comprise the 'cultural interface' (McGloin, 2009).

As researchers, we are acutely aware of the power differentials that can be assumed by university institutions and the conduct of research, thus we wished to provide an example of a successful co-designed Aboriginal community-led research project. Our project adopted a strengths-based approach that identified the enormous skills within the community and draws on the resilience of those communities (Bessarab \& Ng'andu, 2010; Lin, Green, \& Bessarab; 2016; Hunt \& Smith, 2018).Our study assumes that research into matters involving Aboriginal peoples' interests and concerns is best led by Aboriginal researchers, adopt Indigenous research methodologies, ensure Aboriginal intellectual sovereignty, are grounded in Indigenous realities, and include Aboriginal ways of communication (Rigney, 2006).

\section{The Digital Divide and the Non-Indigenous led Initiatives}

As background to the suitability of digital tools that can be used to support Indigenous research methodologies we review the extant literature on Aboriginal people's use of digital technologies the majority of which address (1) digital exclusion as a consequence of the digital divide due to remoteness and/or disadvantage between Indigenous communities and the broader community; (2) the prodigious use of social media for advocacy, mental health and other support systems; and (3) adoption of multimedia tools to preserve and express cultural heritage.

While many Indigenous people live in remote areas of the country and remain digitally excluded from the national communications infrastructure (Australian Bureau of Statistics (ABS), 2017; Australian Communications and Media Authority (ACMA), 2008; Rennie et al., 2019) most of the Indigenous population lives in urban or regional locations. However, even for communities in urban areas, disadvantage can also limit access as depicted in the latest Australian Digital Inclusion Index (https://digitalinclusionindex.org.au/). Despite the belief that Information and Communications Technology (ICT) can overcome the tyranny of distance and disadvantage, the lack of affordable and reliable internet connectivity in Aboriginal communities in Australia remains an issue. For example, a project to provide opportunities for learning in country was compromised by lack of access to technology and inconsistent connectivity (Vodic et al., 2012).

Most literature on digital divides presumes that a material connection to the network society is inherently beneficial, this is not necessarily so for Aboriginal peoples where integration into 
existing systems controlled by state and corporate entities is not in and of itself adequate. For example, Karrippanon (2016) comments that media enabled health interventions are widely used to breach the problems of distance in remote Aboriginal communities, but that little consideration is given to the meaning of the content and to allow a two-way conversation on the material that is presented. As a result, the interventions remain expert driven and top down in their approach despite the democratising potential of the technology.

Due to the cost and technical expertise involved, the creation of the infrastructure needed for rural, regional and remote internet access has been government driven and government funded. Latukefu (2006) reports a case where ICT only appears to remove centralised control from government and into the hands of marginalised communities but, in reality, does not. Latukefu (2006) observes that struggles for self-determination play a more complex sociotechnical role in the drive to bring the information age to Aboriginal communities in Australia.

For over two decades, Community Informatics has been concerned with the use of information and communication technology (ICT) to enable access and assist Aboriginal communities to overcome "digital divides" both within and between communities (Stillman \& Linger, 2009; Gurstein, 2007). While this study promoted the removal of centralized control of ICT out of the hands of government and into the hands of the people (ibid) there is a dearth of research which addresses the core concern of Aboriginal sovereignty and control. Of the few projects that Involve Indigenous people, most early studies published in the 1990s and 2000s, were conducted within a non-Aboriginal paradigm and led by non-Aboriginal researchers.

Other studies show that many Aboriginal Australians have utilised the internet from its early days despite the economic, social, cultural and geographic factors that can affect their access (Rice et al., 2014; Clapham \& Gosden, 2001). In a Canadian study, McMahon (2014) notes how more successful outcomes can be achieved by the "first mile" approach. When the community end is seen as the last-mile, the differences between rural and urban communities are framed as 'problems' or 'shortcomings' to be addressed by simply linking unserved communities to already-existing urban systems and infrastructures. In contrast, the community as the first mile approach implies that decision-making about internet development must be grounded in, and emerge from, the specific needs of local communities. The first mile perspective supports proponents of Aboriginal self-determination where development processes are grounded in a recognition of the inherent laws, institutions, and practices of sovereign Aboriginal peoples (Borrows, 2010).

\section{Digital technologies and community-led initiatives}

In one of the earliest examples of Aboriginal led research into Community Informatics, Turk and Trees (2000) investigated how Aboriginal community processes may be facilitated through the use of information systems which are developed via a highly participative methodology. They describe the successful Ieramugadu Cultural Information System project designed to develop procedures for elicitation, analysis, storage and communication of Aboriginal cultural heritage information with an Aboriginal community in Western Australia.

More recently social media has supported interaction among people who create and share information and ideas in virtual communities and networks. Social media use in Australia has grown rapidly among Aboriginal population with increase in ownership and access to computers, smart phones, tablets and the Internet (Carlson, Farrelly et al., 2015). A review of 
literature on social media and Aboriginal youth in Australia (Rice et al., 2016) provides an insightful overview of its impact on the empowerment of Aboriginal voices. They report that in 2014, 42\% of Australians used Facebook, while Aboriginal and Torres Strait Islander Australians use of Facebook was $60 \%$. Given the mobility of Aboriginal people, Facebook in particular serves as a platform for Aboriginal young people who have moved to reconnect with their Aboriginal identities through online groups where people can share their ideas, thoughts, events, music and photos and through connecting with friends and family. Facebook acts as a modern site for kinship connectivity and continuity and may help reinforce young people's mental health and wellbeing.

An examination of these political and social connections forged via digital tools reveal that Aboriginal movements are grounded in Aboriginal ways of knowing and understanding the world (Wilson et al., 2015). Carlson, Farrelly et al. (2015), for example, reports on a study that examines the ways in which Aboriginal people utilise and interact on social media to assist with suicide prevention strategies. She found that Aboriginal people engage with Facebook to both seek and offer help in relation to preventing suicide and self-harm. She reports that many of the mainstream social risk factors for suicide do not appear to be relevant to Aboriginal people, and that current suicide prevention measures, inappropriately based on western cultural understandings of and attitudes towards suicide and self-harm behaviour and prevention, are therefore ineffective. Carlson's research points to the need to understand Aboriginal suicide in its cultural context, rather than uncritically imposing western notions of health and well-being.

Rice et al. (2016) identified several major themes about how and why Aboriginal people use social media: identity; power and control; cultural compatibility; and community and family connections. The familiarity that many Aboriginal people have with modern technology is reported to give them a sense of fearlessness and control when approaching the use of new platforms. Their confidence in the use of social media suggests that it may also be a useful tool for data collection in research. The multimedia nature of the medium lends itself to the orally and visually focused cultures of Aboriginal communities rather than Western-based literacy. The platforms do not demand a high level of literacy who, with an intuitive understanding of the technology can create content.

Social media provides opportunities for the transmission of Indigenous knowledge across boundaries, for example, knowledge about Aboriginal people for non-Aboriginal people enables the latter to better understand Aboriginal cultures, perspectives and politics. Social media can enable young and old Aboriginal people to reconnect and understand each other better through collaborative efforts between the generations. Kral (2010) comments that through their command of social media, young people are positioned to take a mediating role between cultural knowledge and new digital technologies.

\section{Digital tools for Indigenous research methodologies}

Our review of IS related literature identified a community-based research methodology adopted by Brereton et al. (2014) for a project investigating technology use in a remote Australian Aboriginal community. The approach is based on the concepts of participatory design (PD) and co-design from the field of Human-Computer Interaction (HCI) as an alternative to traditional ethnography. PD relies on partnership with participants in which participants bring essential knowledge of their own context and culture while designers bring 
technical and design facilitation skills creating opportunities for mutual learning and development. A PD approach, also called co-design, can be adopted to facilitate digital data collection by trained community researchers on phone and tablet in the form of text, audio, image or video for studies of Aboriginal communities.

Innovative research dissemination is evident in projects to digitally preserve and communicate Aboriginal knowledge and, with digital empowerment, to "write ourselves back into history." (Bidwell \& Radoll, 2007). Such projects have historically required partnerships between Aboriginal and non-Aboriginal expertise. For example, the Digital History Project, Gugu Badhun people worked with Aboriginal Studies researchers to help design and technologists to implement the interface to their video-history archive. As information systems become easier to use and Aboriginal technical skill grows, Aboriginal technological emancipation will dispense with the divisive praxis of us and them by empowering people as beings for themselves within the process of design (Bidwell \& Radoll, 2007). Through our literature review it was evident a case study approach was needed.

\section{The Case Study Context}

The broad aim of the study was to explore how Aboriginal Community Controlled Organisations (ACCOs) contribute to improving the health and wellbeing of Aboriginal people. To achieve this, we sought to: identify the factors that facilitate the operation of the IKMSG; provide evidence of the effectiveness of its programs, networks and partnerships; examine its impact on the health and wellbeing of the local Aboriginal community; ascertain how it is sustained over time; and use Indigenous research methodologies and theory to interpret this case.

The IKMSG was initiated and is directed by Aboriginal men (the members) living in the Illawarra area with Aboriginal Elders at its helm and supported by the local community. They aim to provide culturally appropriate education programs and services to Aboriginal men and male youth living in the Illawarra, focusing on areas of concern which impacts not only the physical but also the social, emotional and spiritual well-being of the whole community. Two groups of people participated in the research: Aboriginal and non-aboriginal stakeholders, who were nominated by the IKMSG as being part of their organisational network; and IKMSG members. We used mixed methods research that involved conducting a survey and interviewing members of many of the organisations that partner with the IKMSG. Focus groups were also conducted with IKMSG members. Our discussions focused on the way in which Aboriginal people experience and make meaning of their journey through the health system.

The research was conducted in the Illawarra region, a long coastal region located around 70 kilometres south of Sydney, New South Wales, Australia. At the most recent census the region had approximately 8,364 Aboriginal people, making up $2.8 \%$ of the total Illawarra population. The Aboriginal population is a 'young' population, with the average age of Aboriginal people being 21 years of age, compared with 40 years of age in the non-Aboriginal population (ABS, 2017). The ongoing impacts of colonisation in Australia are evident in the multiple levels of disadvantage which continue to be experienced by generations of Aboriginal people across the region, in terms of living in areas of relative socio-economic disadvantage, higher levels of employment, lower levels educational attainment and higher levels of chronic and other health conditions (University of Wollongong Indigenous Multi-Disciplinary Health Research 
Coalition, 2015). This over-representation disadvantage must be understood in terms of the result of historical and ongoing marginalisation of Aboriginal people within the region, rather than as any individual or community 'deficit'. From this standpoint, the fact that Aboriginal people are disproportionately represented in places of high disadvantage, higher unemployment, lower incomes and where children and young people typically experience poorer educational and health outcomes and poorer access to services, is an indictment on the settler state and not on Aboriginal people.

\section{Methodology}

\subsection{Approach and ethical stance}

To ensure the research conformed to these ethical principles for conducting research with Australian Aboriginal peoples (Australian Institute for Aboriginal and Torres Strait Islander Studies, 2012), the research team worked with a project Steering Committee to develop a dissemination plan to ensure that the Illawarra Koori Men's Support Group received feedback on the meaningful results of the research. The researchers acknowledge that all Aboriginal communities are unique and have their own individual set protocols. The Illawarra Koori Men's Support Group gave ongoing support to the researcher from inception to the reporting of results. The researchers acknowledged the wisdom, knowledge and experiences of Aboriginal people living in the Illawarra region. Researchers followed protocols to ensure all stakeholders had an equal voice in the project's governance and operations, and that that any cultural knowledge obtained under this research project would be acknowledged. The researchers also had a full understanding of the responsibilities in undertaking such an Aboriginal community driven research project and provided participants with all necessary information within a safe environment. The research project intended to capture and promote the importance Aboriginal organisations and Aboriginal leadership sustain themselves in the face of complex and challenging social health problems and ongoing policy change.

Ethical approval for the research was obtained from the University Human Research Ethics Committee and the Aboriginal Health and Medical Research Council of NSW (Ethics number 1121/15).

\subsection{Methods}

Faithful to the theoretical underpinning and Aboriginal research approach and following a review of current literature on the role of Aboriginal Community Controlled Organisations, an exploratory mixed-methods case study was conducted over a 12-month period. Data was collected from multiple sources. An online survey stakeholders and social network analysis of 18 organisational representatives was undertaken to investigate the connections of the IKMSG with other organisations within the region. Semi-structured interviews and focus group discussions with members of the IKMSG (men enrolled in IKMSG programs) were conducted by Aboriginal researchers who were sensitive to the ethical considerations described above. We also worked collaboratively with group coordinators on the co-development of website and social media.

\subsection{Qualitative Analysis}

All of the qualitative data collected from the interviews and focus groups was entered into NVivo Software (QSR International Pty Ltd, 2012). A project was set up in the NVivo computer program and interview transcripts were imported into the program along with researcher 
notes and observations program data, documents and literature. The audio recorded interviews were transcribed professionally. Interview transcripts and tapes were securely stored on password protected storage space on the researchers' server.

For the analysis of the semi-structured interviews, the transcribed interviews were initially auto coded based on the direct question asked in the interview schedule, and then analysed thematically to provide a more grounded analysis of this data. Based on an initial analysis of the answers to this question a framework was developed for ongoing coding. The initial coding framework was around the following codes: Emergence; Current Operations; Community Context; Programs; Clients; Sustainability; Networks; Relationships; Values; Community Control; and Leadership.

For the three focus groups a thematic analysis was undertaken exploring the personal experiences of members' participation in group programs and activities across the broad areas of enquiry (getting involved; transforming men's lives; impact on community; and collaboration and partnerships).

\subsection{Social Network Analysis}

SNA is a method of mapping and analysing networks of organisations or individuals. The patterns of interaction are explored to explain the engagement between organisations or individuals in the network including where the concentrations of trust and power lie. In this study the IKMSG's inter-organisation network is examined by means of 1) a visual analysis and 2) SNA measures.

In the visual analysis, sociograms are used to map the organisations which have relationships (links) with the organisation being studied and to show how these organisations are linked. Nodes (circles or squares) represent the organisations in the network, the lines represent the links (relationships), and the arrows show the direction of relationship in the network. Lines contain information about which organisations initiate and receive transactions, such as funding. For example, funding may be given by Organisation A and received by Organisation B.

The primary measures used in the study to explore the interactions between organisations are all related to centrality. Centrality tells us who and how important the organisation is in the network. The three centrality measures used to analyse the network are degree, between-ness and closeness as follows.

Degree centrality are connectors (hubs) in the network identified as those organisations with links to the greatest number of organisations in the network. Degree centrality indicates status and trust and is calculated as the number of links/ties as a proportion of the number of organisations in the network In-degree and out-degree count the number of relationships to and from organisations, respectively (Freeman, 1979).

In-degree (connected-trusted) is used to identify connectors (hubs) and measure collaboration in a network (Valente, 2010). A high in-degree indicates that an organisation plays an important role in the network (Valente, 2010). They are the most trusted for information and partnerships (namely "funding", "service delivery" and "culture") (Long et al., 2013). A low in-degree would indicate that an organisation is connected to few organisations in the network. 
Out-degree (connected-influential) measures the number of links (relationships) directed away from an organisation in the network (Freeman, 1979). A high out-degree indicates an organisation is connected (linked) to many organisations, and a low out-degree would indicate that an organisation has few links. Organisations who have unusually high out-degree are organisations that are able to exchange with many others or make many others aware of their views (Valente, 2010; Long et al., 2013). For this reason, they are often said to be influential diffusers of knowledge, attitudes and behaviours (Hanneman \& Riddle, 2005).

Betweenness centrality Powerful organisations in the network were identified as those organisations who were acting as gatekeepers or brokers of knowledge, attitudes and behaviour (Long et al., 2013). Betweenness measures the extent to which an organisation lies on the shortest path (geodesic distance) to organisations that are not connected (Freeman, 1979). The greater the betweenness centrality the more control that organisation has in passing and sharing information. Brokers can add substantive value to networks by generating new ideas and making advice and knowledge more accessible to others. Broker organisations act as gatekeepers controlling the information passing into and out of their group or network (Long et al., 2013). They may hoard information, but they are generally positively associated with diffusing useful information (Long et al., 2013).

Closeness centrality (Influential) Closeness centrality is a measure of the average shortest distance from each organisation to each other organisations (Freeman, 1979). A lower closeness centrality score indicates a more central therefore important position as a diffuser of knowledge, attitudes and behaviours in the network. A closeness centrality score is equal to one would indicate an organisation is directly connected to everyone in the network, good position to initiate the diffusion of information through the network. Centrality scores indicate how quickly an organisation can interact and communicate with others in the network of knowledge, information and/or resources (Borgatti, 2005).

The study used an ego-centric model which looks at all relationships in a single bounded network of organisations (Hawe, Webster, \& Shiell, 2004). Data collection was conducted through an online survey. Ucinet 6TM and Netdraw 2.138TM were used to compute and analyse the data and create the network maps.

\begin{tabular}{|l|lll|l|l|l|}
\hline $\begin{array}{l}\text { Organisations }(\mathrm{n}=24)^{*} \\
\text { Type of organisation }\end{array}$ & Sector & & Target population & \\
\hline Aboriginal organisation & 7 & Education & 6 & Non-aboriginal & 11 \\
\hline Government organisation & 11 & Employment & 3 & Aboriginal population & 13 \\
\hline Non-Government organisation & 4 & $\begin{array}{l}\text { Families and } \\
\text { Community }\end{array}$ & 6 & & \\
\hline Higher education & 2 & Health & 5 & & \\
\hline & & Justice & 3 & & \\
\hline & & Housing & 1 & & \\
\hline
\end{tabular}

Table 1. Characteristics of organisations represented in the study

Note: The number of organisations was 24; the divisions of a number of large organisations were included. 


\section{Results}

\subsection{Participants}

Thirty-six people were identified by IKMSG as stakeholders and were subsequently approached to participate in the study. These stakeholders were based on the IKMSG's current and past organisational relationships. A total of 21 stakeholders participated in the research by completing a survey and/or semi-structured interview (58\%). Of the stakeholders who did not participate, three indicated that their organisation was already represented by another survey respondent, nine did not respond to the email and phone invitations; one person was no longer employed by the organisation, and two declined to participate. Table 1 describes the demographic characteristics of the 21 stakeholders who participated in the study.

A total of 24 organisations or divisions of organisations (including IKMSG) were represented by the survey respondents and included in the network. As seen in Table 1, these organisations provide services across a wide range of sectors within the Illawarra region.

\subsection{Results: Social Network Analysis}

In the SNA network four organisations (nodes) were ACCOs, five were non-government organisations and the remaining were government organisations.

The types of connections listed were: having a memorandum of understanding (MOU); funding relationship; worked together on projects; working together on meetings; and cultural connections. These relationships were either one way or reciprocal. All members of the network were engaged in projects and all but one were involved in meetings. There were twelve MOUs between organisations. There were 11 organisations involved in cultural activities and nine organisations engaged in the exchange of funding.

The most relevant results for this study are now shown. These are in-degree centrality, which is an indication of trust, and between-ness, which is an indication of power/and what?

\subsubsection{In-Degree Centrality}

Degree centrality is a measure of the links an organisation has to other organisations in the network. In-degree counts the number of relationships to an organisation. From Figure 1 (below) we can see three of the four ACCOs including the IKMSG have the most connections in the network being highly trusted and respected organisations.

\subsubsection{Between-ness Centrality}

Between-ness centrality is measured by the number of times an organisation acts as a path between two organisations. Figure 2 (below) shows between-ness centrality with the largest square, IKMSG, having the highest between-ness centrality in the network.

The study found that ACCOs were highly involved in all activities which were part of the network. The ACCOs were also highly networked as is demonstrated by their central location in the network and by being visually displayed as larger players in the measurements of degree, between-ness and closeness centrality measures. Figures 1 and 2 show the prominence of the IKMSG in the SNA maps suggesting that its work is highly respected in both Aboriginal and non-Aboriginal local organisations giving validity to the model produced by the qualitative analysis. 
The SNA revealed that the IKMSG was central in the provision of a wide range of social, health-related and cultural activities. However, despite all the high activities within the network by ACCOs, there was little funding directed to these organisations compared to nongovernment organisations within the network.

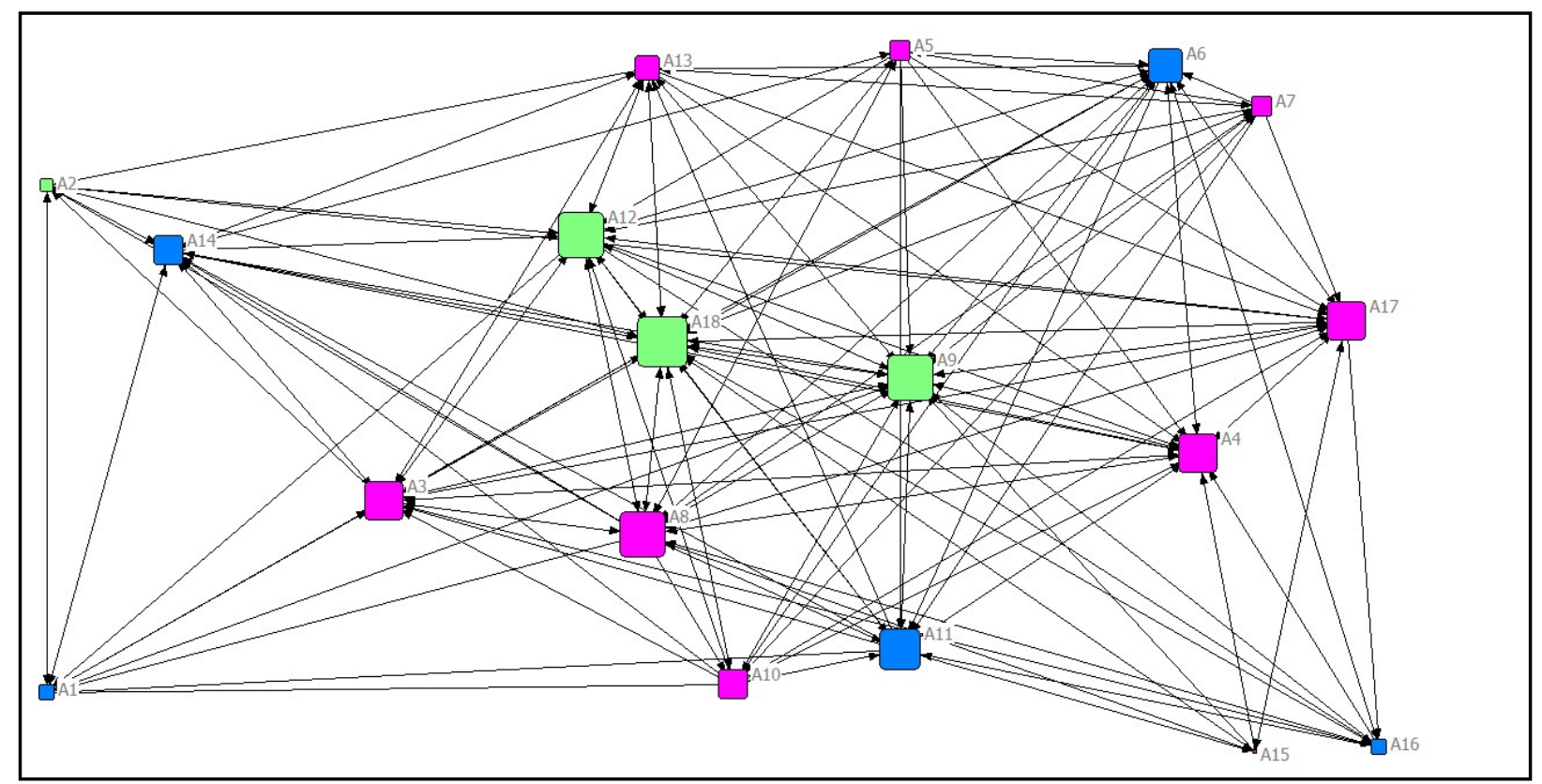

Figure 1. Network map measuring degree centrality
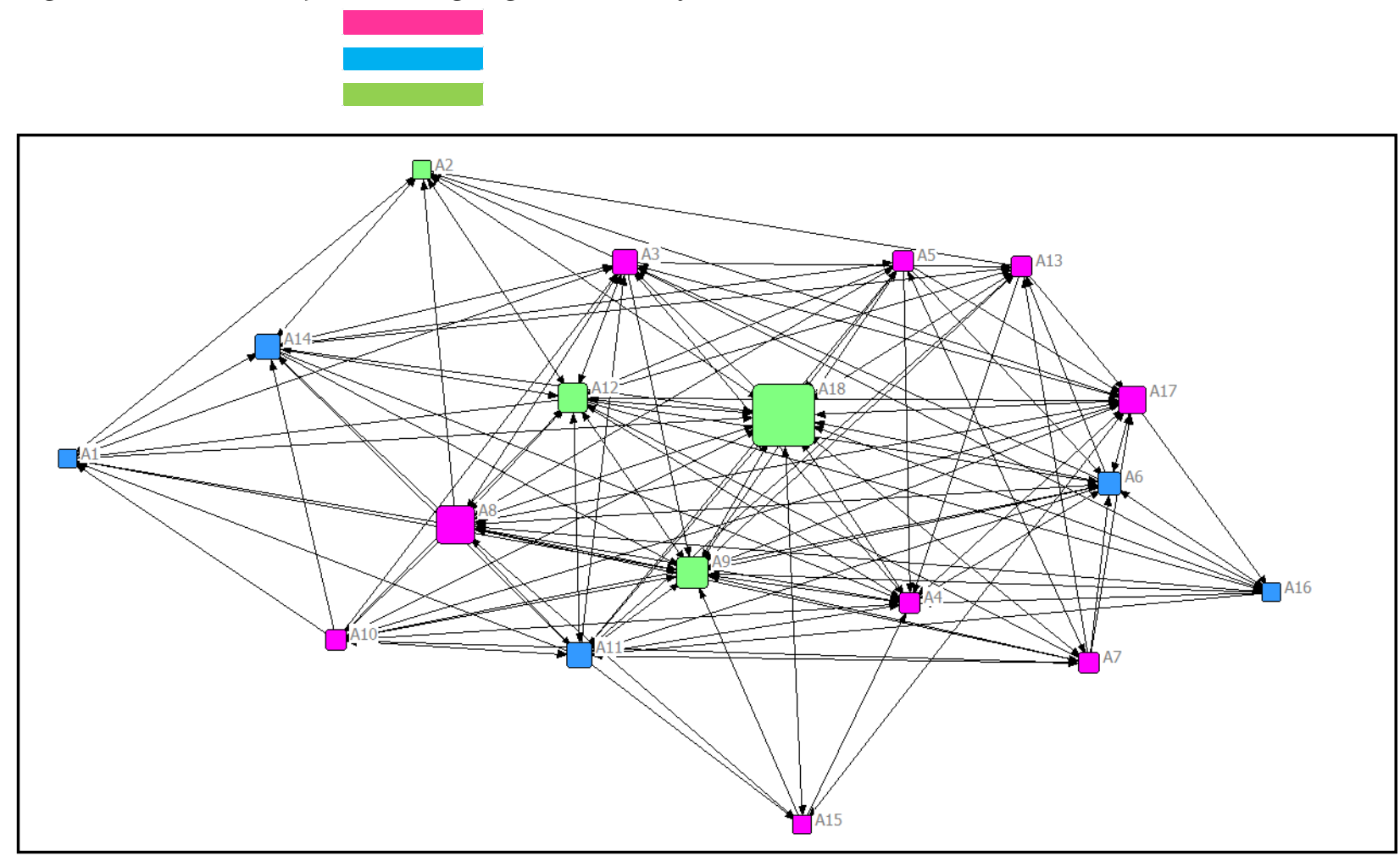

Figure 1. Between-ness Centrality 


\subsection{Results: Knowledge Management and Dissemination}

Prior to our project commencing University of Wollongong students worked with the IKMSG to set up a Facebook page and trained one member of the group to administer the page. When used in this manner, Facebook becomes a tool that instantiates Aboriginal Standpoint Theory (Nakata, 2007; Foley, 2003; Carlson, Jones et al., 2015) and the concept of the Cultural Interface. Rice et al (2016) have reported how Aboriginal young people have embraced social media such as Facebook to produce their own unregulated content with limited technical skills but an intuitive understanding of what the technology affords in allowing them to tell their own story.

An early meeting between the IKMSG and the research team identified the value of having an online space for knowledge transfer between the IKMSG, the researchers and the community. It was decided that one of the researchers would work with one member of the IKMSG to create a website for the IKMSG. Following the success of the Facebook page, the content of the page would be created and maintained by members of the IKMSG. All agreed on different and distinct roles for the Facebook page and the website. Facebook would continue to post photos and news of IKMSG events. The Website would describe the different projects and activities of the IKMSG to the community, which included local Aboriginal and nonAboriginal audiences, and present a positive image to potential sponsors of the IKMSG projects where funding had become a real problem. In turn the researchers set up their own blog as one way to transfer knowledge on the outcomes of the research to the non-academic community.

Leveraging the strong partnership between the IKMSG and the research team, the IKMSG Website and Facebook page were co-developed and maintained throughout the project. The activity of the IKMSG Facebook page consists of posts of labelled videos and images of significant activities of the group with 266 followers among the community. Since the page was established 2.5 years ago 6 videos have had 415 views an average over 200 likes each. 250 photographic images have been posted with over 1000 likes. While there is little other activity, the regular postings provide a means by which the community shares knowledge of current events. (See https://www.facebook.com/IKMSG)

The website has been live for almost two years and contains information about the IKMSG and its regular ongoing programs and provides useful health, social and other information to its members and the broader community. There is little need to update the page which is only done on a 6-monthly basis.

On the research side a blog was set up on the University Website providing updates to research projects and providing access to both Community and Research Reports

\subsection{Results: The ACCO model}

In the Introduction to this paper, we reported that the results of the study show (1) how ACCOs can leverage the knowledge inherent in communities to build resilience and create better futures; and (2) how real participation and inclusive decision making, provide the authority that enables change.

Qualitative analysis of interviews, supported by the findings of the SNA, show that the IKMSG has operated successfully for the past ten years. In the 2016-2017 reporting year a total of 2770 people attended the IKMSG and 21 local services regularly drew on its programs. Over the 
past decade the group has gone from strength to strength, and it is one of the most highly respected Aboriginal organisations in the region.

The Group is guided by a clearly articulated set of principles and values. It has strong leadership and has developed a governance structure which places a high value on respect, the role of Elders, and commitment to developing programs in response to community need. The model emanating from this research provides evidence for the multiple benefits of the strong presence of Aboriginal Elders as advisors, role models for young men and sources of cultural knowledge within the education system.

The respect that people have for the Elders has a positive impact all members of the men's group. While a full presentation of the results of the qualitative research is published elsewhere (Ngarruwan Ngadju, 2017a) the following words of one participant is compelling:

I think when you see the Uncles out and they walk around, it shows a lot of honour as well, like honour towards - like we're respectful towards our women, respectful towards our culture and, you know, it shows the wider view of what we want to present ourselves as honourable men out in the community and I think we bring a lot of honour and respect to the Uncles that are here, and the young generation. (Focus group participant)

The research has identified ten key elements of success which underpin the IKMSG model as shown in Figure 3 (below).

The depiction of the dominant presence of the IKMSG on the SNA map was interpreted by members of the community as giving authenticity to this model as a successful and influential ACCO, IKMSG is uniquely able to provide a range of unique culturally focused services for the Aboriginal community in the Illawarra. The success of IKMSG in this important work is directly due to its identity as an ACCO. These services cannot be replicated by mainstream organisations. The IKMSG is a part of the world leading efforts of ACCOs to deliver best practice services that are underpinned by principles of self-determination.

\section{Discussion and Conclusion}

This paper addresses issues of concern to local Aboriginal communities. The investigation was led by indigenous researchers in partnership with community participants. Indigenous participatory research methodologies were adopted and supported by suitable digital tools so that the investigators had access to the knowledge embedded within the Aboriginal communities. This gives us confidence in the quality of the collected data and its analysis, leading to findings of authentic theoretical and practical value.

The use of digital tools in an interdisciplinary context provided a unique lens and led to findings that were otherwise unavailable. Importantly they provided a way of understanding the organisation in a way that had meaning for the organisation and the researchers. What is it about these digital tools that provides information that is unique and use for this organisation? Returning to the previously referenced principles of Indigenous research methodologies identified by Martin (2003), this research project recognised Aboriginal worldviews, knowledge and realities as distinctive and vital to Aboriginal existence and survival, as a research framework in this study.

The SNA analysis provides an example of the roles that Information Systems can play to support the conduct of research in Aboriginal communities. Social networks are deeply 
embedded in Indigenous society and Indigenous knowledge; relationships, reciprocity and respect are central to the way Indigenous communities relate to each other. In many ways the SNA validated what was already known; importantly though, it provided a visualisation of networks and relationships which replicates the modes of communication and reciprocal networks that are so fundamental to the way Aboriginal organisations work. But the SNA analysis also highlighted the large discrepancy between the longstanding networks and the relative lack of economic support for the organisation.

\section{Leadership}

Leadership from a group of passionate, well respected and well-known Elders and Aboriginal community leaders has enabled the 'coming together' of men from many walks of life.

\section{Key personnel}

Key personnel (Board Members, Staff and Volunteers) have the requisite skills and capacity to design, develop and implement programs.

\section{Strong values-based ethos}

A strong, values-based ethos of trust, support, confidentiality and of being role models for the younger generation, underlie the organisation.

\section{Holistic approach to service delivery}

The holistic approach to service delivery is characterised as 'respectful', 'interactive', 'holistic', 'community based', 'healing' and 'inclusive'.

\section{Culturally safe}

The group provides a model for how to deliver culturally safe and meaningful programs that strongly resonate with the target audience.

\section{Effective responsive programs}

Effective programs respond to areas of critical need and provide members with resources to look after their physical, emotional and social health and wellbeing.

\section{A Unique pedagogy}

A unique 'IKMSG pedagogy' describes the group's capacity and skill in culturally engaging and developing healing relationships with Aboriginal men.

\section{Highly connected}

The group is highly connected through its partnerships with a broad range of services and agencies within the region.

\section{Reconnecting men}

The group provides a much needed mechanism for reconnecting men with the Aboriginal community.

\section{Elders and education}

Aboriginal Elders have a strong presence in the education system as advisors, role models for young men and sources of cultural knowledge.

\section{Figure 3. The model of a successful ACCO emerging from the IKMSG study}

Overall, the research revealed the unique role of the IKMSG in holistic service provision and support for Aboriginal men and youth in the Illawarra region. The holistic and culturally safe programs respond effectively to areas of critical need in health, education, justice and employment. The strong presence of Elders in the educational programs has created a unique pedagogy with capacity and skill in culturally engaging and developing healing relationships with Aboriginal men and reconnects them to community. Through its strong networks and well-established partnerships with a broad range of organisations the group has a powerful 
influence in promoting respect for Elders amongst both Aboriginal and non-aboriginal people within the local Illawarra community.

The strong consistent leadership from Aboriginal Elders and effective management are key factors in the success of the IKMSG over the past decade. The IKMSG model this research has developed offers a form of engagement which could benefit many other First Nations communities. The Elders are vitally important to the Group and are recognized and highly utilised by government and community organisations for their expert cultural knowledge and community contacts. The research honoured Aboriginal social mores as essential processes through which members of the IKMSG live, learn and situate themselves in their own lands and in the lands of other Aboriginal peoples. It emphasised the social, linguistic, cultural, historical and political contexts which shape Aboriginal experience, lives, positions and futures through the development of the IKMSG model. It strove to privilege the voices, experiences and lives of Aboriginal people and Aboriginal lands throughout the conduct of the research.

We put this model forward as an instance of a successful ACCO for others to follow and is accepted as such by the community. The ACCO model confirms the critical importance to beneficial outcomes and sustainability that comes through Aboriginal-community control of services provided within local Aboriginal communities. This model is interpreted through the four Aboriginal theoretical principles beginning with the recognition of the Aboriginal worldviews, knowledge and realities as distinctive and vital to Aboriginal existence and survival and consistently privileging the voices, experiences and lives of Aboriginal people and Aboriginal lands. The results of this research demonstrate the futility of ignoring the Aboriginal First Nation's sovereignty and benefits of acknowledging their right to be included in decision-making that impacts Aboriginal futures. Empowering local Aboriginal communities provides a mechanism for change. It disrupts the deficit mindset that frames Aboriginal Australians as undeserving and complicit in their disadvantage, to one of resilience and strength.

\section{Acknowledgements}

We would like to acknowledge the Uncle Gerald Brown, Mr Gregg Potts, and Mr Michael Willis of the Illawarra Koori Men's Support Group, for their ongoing support and collaboration through our research collaboration. We also acknowledge Dr Scott Winch for his contribution to original research on which this paper is based.

\section{References}

ABS Australian Bureau of Statistics. (2017) Census: Aboriginal and Torres Strait Islander population. http://www.abs.gov.au/ausstats/abs@.nsf/mediareleasesbyReleaseDate/ 02D50FAA9987D6B7CA25814800087E03?OpenDocument. Retrieved 17/12/2018.

Australian Communications and Media Authority. (2008). Telecommunications in remote Indigenous communities. Canberra.

Armstrong, A., Buckley, S., Londsdale, M., Milgate, G., Kneebone, L.B., Cook, L. \& Skelton, F. (2012). Starting School: A Strengths-based Approach. Towards Aboriginal and Torres Strait Islander Children, Australian Council for Educational Research. Canberra. 
Australian Institute for Aboriginal and Torres Strait Islander Studies. (2012). Guidelines for Ethical Research in Australian Indigenous Studies. Canberra, AIATSIS.

Bessarab, D., Green, S., Jones, V., Stratton, K., Young, S. \& Zubrycki, J. (2014). Getting it Right Creating Partnerships for Change. Integrating Aboriginal and Torres Strait Islander knowledges in social work education and practice. Teaching and Learning Framework 2014. Office for Teaching and Learning: Australian Government.

Bessarab, D. \& Ng'andu, B. (2010). Yarning About Yarning as a Legitimate Method in Indigenous Research. International Journal of Critical Indigenous Studies, 3(1).

Bidwell, N. J., \& Radoll, P. (2007). Redisplacement by design. Interactions, 14(2), 12-14.

Borgatti, S.P. (2005). Centrality and network flow. Social Network, 27, 55-71.

Borrows, J. (2010). Canada's Indigenous constitution. Toronto, Canada: University of Toronto Press.

Brereton, M., Roe, P., Schroeter, R. \& Lee Hong, A. (2014). Beyond ethnography: engagement and reciprocity as foundations for design research out here. In Proceedings of the SIGCHI Conference on Human Factors in Computing Systems (pp. 1183-1186). ACM.

Carlson B., Jones L., Harris M., Quezada N. \& Frazer R. (2015). Trauma, Shared Recognition and Indigenous Resistance on Social Media, Australasian Journal of Information Systems, 21.

Carlson B., Farrelly T., Frazer R. \& Borthwick F. (2015). Mediating Tragedy: Facebook, Aboriginal Peoples and Suicide, Australasian Journal of Information Systems, 19, Special Issue on Research on Indigenous ICT.

Clapham, K. \& Gosden D. (2001). Education and Indigenous Health in a Globalised World. AARE, Fremantle.

Denzin, N. K. \& Lincoln, Y. S. (2008). Handbook of critical and Indigenous Methodologies. Thousand Oaks, California: Sage.

Fforde, C., Bamblett, L., Lovett, R., Gorringe, S. \& Fogarty, B. (2013). Discourse, Deficit and Identity: Aboriginality, the Race Paradigm and the Language of Representation in Contemporary Australia. Media International Australia, 149, 162-173.

Fogarty, W., Bulloch, H., McDonnell, S., \& Davis, M. (2018). Deficit Discourse and Indigenous Health: How Narrative Framings of Aboriginal and Torres Strait Islander People Are Reproduced in Policy. Deficit Discourse and Indigenous Health: How Narrative Framings of Aboriginal and Torres Strait Islander People Are Reproduced in Policy, xii.

Foley, D. (2003). Indigenous Epistemology and Indigenous Standpoint Theory Social Alternatives 22(1): 44-52.

Fredericks, B.L. (2009). The epistemology that maintains white race privilege, power and control of Indigenous Studies and Indigenous peoples' participation in universities. Australian Critical Race and Whiteness Studies Association eJournal, 5(1), 1-12.

Fredericks, B., Adams, K., Finlay, S., Fletcher, G., Andy, S., Briggs, Lyn, Briggs, L. \& Hall, R. (2011). Engaging the Practice of Indigenous Yarning in Action Research. Action Learning Action Research Journal. 17 (2), 9-20. 
Fredericks, B., Longbottom, M., McPhail-Bell, K. \& Worner, F. in collaboration with the Board of Waminda. (2016). Dead or Deadly Report. Waminda Aboriginal Women's Health Service. CQ University, Australia: Rockhampton, 32 pages, ISBN: 9781921047169. Access at: http://acquire.cqu.edu.au:8080/vital/access/manager/Repository/cqu:13773

Freeman, L.C., Roeder, D. \& Mulholland, R. R. (1979) Centrality in social networks: II. Experimental results. Social Networks, 2(2), 119-141.

Gorringe, S., Ross, J. and Fforde, C. (2011). Will the Real Aborigine Please Stand Up?': Strategies for Breaking the Stereotypes \& Changing the Conversation. AIATSIS Discussion Paper 28. Canberra, AIATSIS.

Gurstein, M. (2007). What is Community informatics? (And Why Does It Matter), Polimetrica, Milan.

Hanneman, R.A., Riddle, M. (2005). Introduction to Social Network Methods. University of California.

Hawe, P., Webster, C. \& Shiell, A. (2004). A glossary of terms for navigating the field of social network analysis. Journal of Epidemiology and Community Health, 58(12), pp. 971-975.

Hollinsworth, D. (2013). Decolonizing indigenous disability in Australia. Disability \& Society, 28(5), 601-615.

Hunt, J. \& Smith, D. E. (2018). Building Indigenous community governance in Australia: Preliminary research findings. Canberra, ACT: Centre for Aboriginal Economic Policy Research, Research School of Social Sciences, College of Arts \& Social Sciences, The Australian National University. University, National Centre for Indigenous Studies.

Karripanon, K, (2016). A double edged spear: the social life of youth, mobile phones and social media in a remote Aboriginal community, University of Wollongong PhD Thesis.

Kral, I. (2010). Plugged in: remote Indigenous youth and digital culture, CAEPR working paper No 69/201, Australian National University, Canberra.

Latukefu, A. S. (2006). Remote Indigenous communities in Australia: Questions of access, information, and self-determination. In K. Landzelius (Ed.), Native on the net: Indigenous and diasporic peoples in the virtual age (pp. 43-60). London, UK, and New York, NY: Routledge.

Lin, I., Green, C. \& Bessarab, D. (2016). 'Yarn with me': applying clinical yarning to improve clinician-patient communication in Aboriginal health care. Australian Journal of Primary Health. doi:10.1071/PY16051

Long, L.C., Cunningham F.C. \& Braithwaite J. (2013) Bridges, brokers and boundary spanners in collaborative networks: a systematic review. BMC Health Service Research, 13. https://doi.org/10.1186/1472-6963-13-158

Martin, K. \& Mirraboopa, B. (2003). Ways of Knowing, Being and Doing: A Theoretical Framework and Methods for Indigenous and Indigenist Re-search. Journal of Australian Studies, 27, 203-214.

Martin, K. L. (2008). Please knock before you enter: Aboriginal regulation of outsiders and the implications for researchers, Teneriffe, Queensland, Post Pressed. 
McCallum, K., Waller, L. \& Meadows, M. (2012). Raising the volume: Indigenous voices in news media and policy. Media International Australia, 142(1), 101-111.

McGloin, C. (2009). "Considering the work of Martin Nakata's "Cultural Interface": a reflection on Theory and Practice by a Non-Indigenous Academic." The Australian Journal of Indigenous Education, 38, 36-41.

McMahon, R. (2014). From digital divides to the first mile: Indigenous peoples and the network society in Canada. International Journal of Communication, (8), 25.

Martin, K. (2003). Ways of Knowing, Being and Doing: A Theoretical Framework and Methods for Indigenous and Indigenist Re-search. In K. McWilliam, P. Stephenson, \& G. Thompson (Eds.), Voicing dissent, New Talents 21C: Next generation Australian Studies (Vol. 76, pp. 203-214). St Lucia: University of Queensland.

Moreton-Robinson, A. (2000). Talkin'up to the white woman: Aboriginal women and feminism, Univ. of Queensland Press.

Nakata, M. (2007). The Cultural Interface. The Australian Journal of Indigenous Education, (36), 7-14.

Ngarruwan Ngadju (2017a). Interdisciplinary Indigenous Health Research Group in collaboration with the Illawarra Koori Men's Support Group Caring for Community Research Report: Valuing the significant contribution of the Illawarra Koori Men's Support Group. University of Wollongong.

Ngarruwan Ngadju (2017b). Interdisciplinary Indigenous Health Research Group with the Illawarra Koori Men's Support Group. Caring for Community: Community Report. University of Wollongong

QSR International Pty Ltd. (2012). NVivo qualitative data analysis software; Version 10 (Version 10).

Rennie E., Thomas J. \& Wilson C. (2019). Aboriginal and Torres Strait Islander people and digital inclusion: what is the evidence and where is it? Communication Research and Practice, 5:2, 105-120.

Rice, E. S., Haynes, E., Royce, P. \& Thompson, S. C. (2016). Social media and digital technology use among Indigenous young people in Australia: a literature review. International journal for equity in health, 15(1), 81.

Rigney, L.-I. (2006). Indigenist Research and Aboriginal Australia. In N. Goduka, I \& Kunnie, J. (Ed.), Indigenous People's Wisdoms and Power: Affirming Our Knowledges through Narrative (pp. 32-50). London: Ashgate Publishing.

Sarra, C. (2011). Strong and Smart - Towards a Pedagogy for Emancipation: Education for First Peoples, New York, Routledge.

Smith, L. T. (2012). Decolonizing methodologies: Research and Indigenous peoples. (2nd Edition) Zed Books Ltd. (1st Edition 1999)

Stillman, L., Linger, H. (2009). Community Informatics and Information Systems: how can they be better connected? The Information Society 25(4), 255-264. 
Turk, A. \& Trees, K. (2000). Facilitating community processes through culturally appropriate informatics: An Australian indigenous community information systems case study. In Community Informatics: Enabling Communities with Information and Communications Technologies (pp. 339-358). IGI Global.

University of Wollongong Indigenous Multi-Disciplinary Health Research Coalition (2015). Illawarra Aboriginal Community Profile: a snapshot of an urban Aboriginal community, University of Wollongong, Wollongong.

Valente, T. W. (1996). Social Network thresholds in the diffusion of innovations. Social Network, 18(95), 69-89. https://doi.org/10.1016/0378-8733(95)00256-1

Vigo, K. G. M. (2017). One country: different voices-meeting the challenge of introducing Indigenous approaches to learning to non-Indigenous students. Creative work and exegesis. https://researchbank.swinburne.edu.au/file/92924766-2378-43d9-a4ae05d8bccb8865/1/kitty_vigo_thesis_1.pdf

Vodic, A., Senior, K., Dwyer, B. \& Szybiak, P. (2012). A remote chance? The potential for Indigenous students to participate in online learning in remote communities in the Northern Territory, Charles Darwin University, Darwin.

Walker, M., Fredericks, B., Mills, K. and Anderson, D. (2013). 'Yarning' as method and process for community-based health research with Indigenous Women. The Indigenous Women's Wellness study. Healthcare for Women International, 00, 1-11: 2013.

Walter, M. \& Andersen, C. (2013). Indigenous Statistics: A Quantitative research methodology, New York, Routledge.

Walter, M. \& Moreton-Robinson, A. (2010). Indigenous methodologies in social research (Online Chapter 22). In M. Walter (Ed.), Social Research Methods (2nd Edition), pp. 1-18. Australia: Oxford University Press.

Walter, M. \& Suina, M. (2018). Indigenous data, indigenous methodologies and indigenous data sovereignty. International Journal of Social Research, DOI:10.1080/ 13645579.2018.1531228.

Williams, H., Hunter, K., Griffin, B., Kimble, R. \& Clapham, K. (2021). Fire and smoke: Indigenous research methodologies exploring psychosocial impact of paediatric burns on Aboriginal and Torres Strait Islander families. International Journal of Qualitative Methods, 20, pp. 1-14.

Wilson A., Carlson B. \& Sciascia, A. (2015). Reterritorialising Social Media: Indigenous People Rise Up, Australasian Journal of Information Systems, vol. 19, Special Issue on Research on Indigenous ICT.

Wilson, S. (1998). Research Is Ceremony: Indigenous Research Methods. Fernwood Publishing. 
Copyright: (C) 2021 authors. This is an open-access article distributed under the terms of the Creative Commons Attribution-NonCommercial 3.0 Australia License, which permits noncommercial use, distribution, and reproduction in any medium, provided the original author and AJIS are credited.

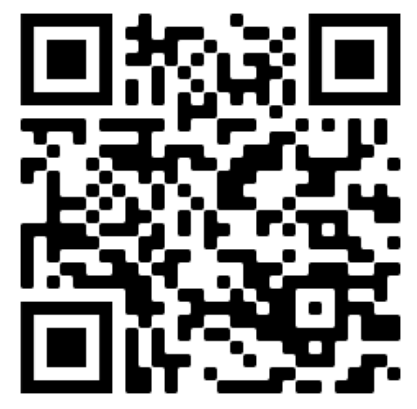

